\title{
(1) \\ PROTECTING IMPORTANT STRUCTURES AGAINST MAN- MADE HAZARDS USING THE CONVEX DOTTED TECHNIQUE \\ Analysis and simulation using the AUTODYN simulation package
}

\author{
EHAB HANAFI MAHMOUD* \\ MOHAMED ALI BARAKAT**
}

\begin{abstract}
In the light of recent terrorists' attacks on facilities throughout the world, planners, architects and engineers are beginning to re-visit conventional approaches in the design of high-security facilities. Increasingly, existing the strategic installations are being protected to survive a limited threat. These actions are essential to mitigate the hazardous effects of explosions and impact. Important facilities are made to a generally higher degree of robustness than conventional installations.

Protection can be provided by provision of a massive outer structure or by ensuring large stand-off distance. Given the typical location of installations such as embassies, government and other office buildings, which could be subjected to terrorist attack, at the heart of major cities, it is not normally possible to ensure appropriate stand-off distance. A massive outer structure may be too expensive and inconsistent with the functionality and image of the installations.

The Architect has a contribution to make in the enhancement of structural survivability by optimizing the configuration (shape, form, elevation, cross section, etc.) to provide a measure of protection without compromising appearance and utility.

This paper presents the findings from a program of research, which explores the opportunities offered by an effective architectural approach. A group of scenarios simulated and examined by analysis the concept of using new protection techniques, as assessed using the AUTODYN computer simulation package in 2D \& 3D V4.[1]
\end{abstract}

\section{KEYWORD}

Architecture, Protection, Blast, Structure, Hazard.

\footnotetext{
* Permanent staff (MSc.,PhD) - Architecture |Department. Military Technical Collage - Cairo, EGYPT

** Permanent staff (MSc.,PhD) - Architecture IDepartment. Military Technical Collage - Cairo, EGYPT
} 


\section{REFERENCE SCENARIO}

The Reference scenario, with which all simulations will be compared, is an explosion in front of a structure as shown in figure 1 . The AUTODYN simulation package 2D \& 3D V4 was used to evaluate the pressure and impulse values on the structure and the ConWep program [2] validated these values [Figure 2].

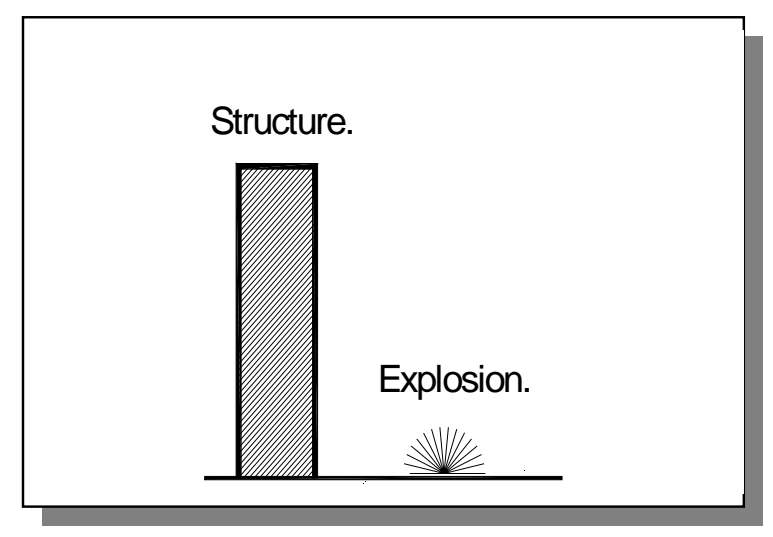

Fig. 1 Reference Scenario.

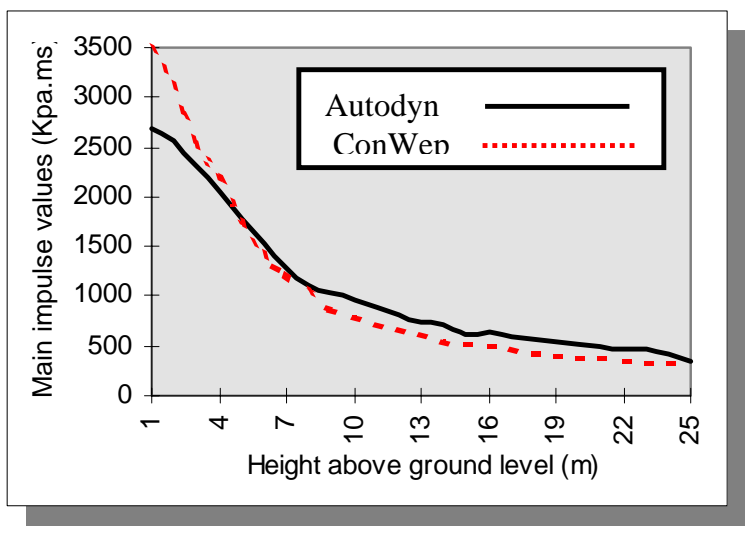

Fig. 2 Impulse values of the Reference Scenario

\section{Parameters and limits of simulations}

Several scenarios were prepared to represent typical geometries, taking into consideration the following variables: [Figure 3]

1. Distance of the structure from the blast.

2. Target structure height.

3. The dotted elevation configuration. 


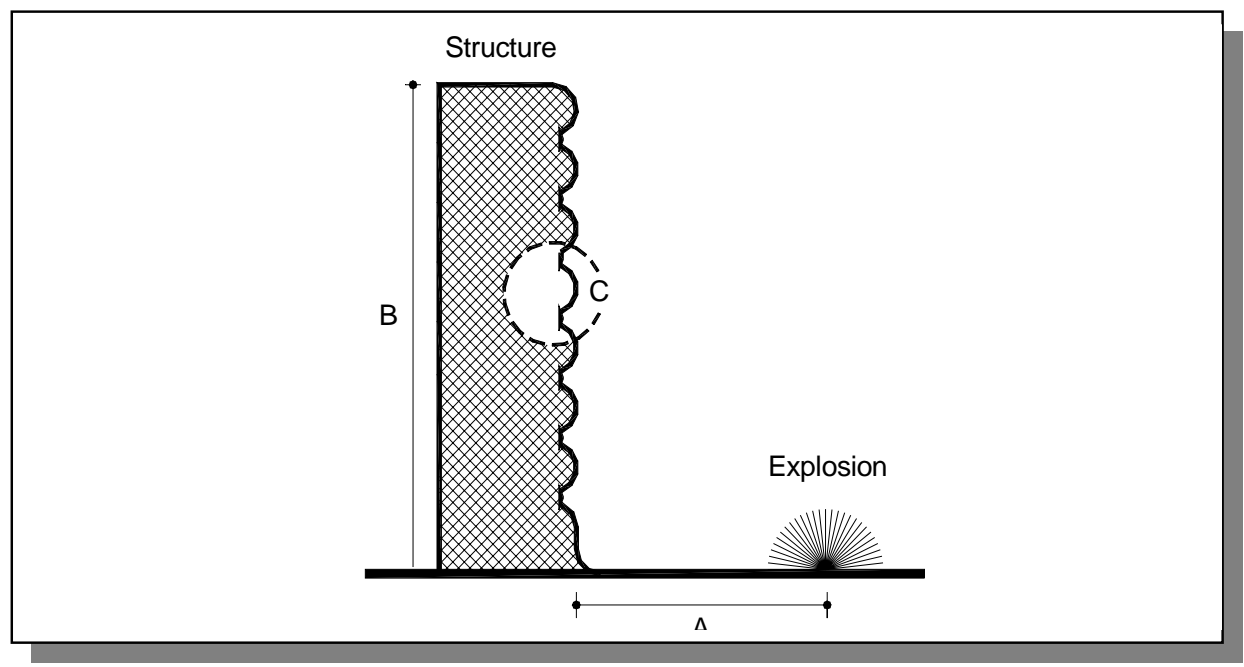

Fig. 3 Parameters and Limits of Simulations.

\section{Distance of the structure from the blast}

The worst case scenario was taken to be when a vehicle bomb is located at a stand-off of $5 \mathrm{~m}$ from the structure, as shown in figure 4 . This was based on: [3]

1. An assumed minimum pedestrian path width of $3 \mathrm{~m}$.

2. An assumed planting zone width of $1 \mathrm{~m}$.

3. 1m distance between center of explosion and the planting zone.

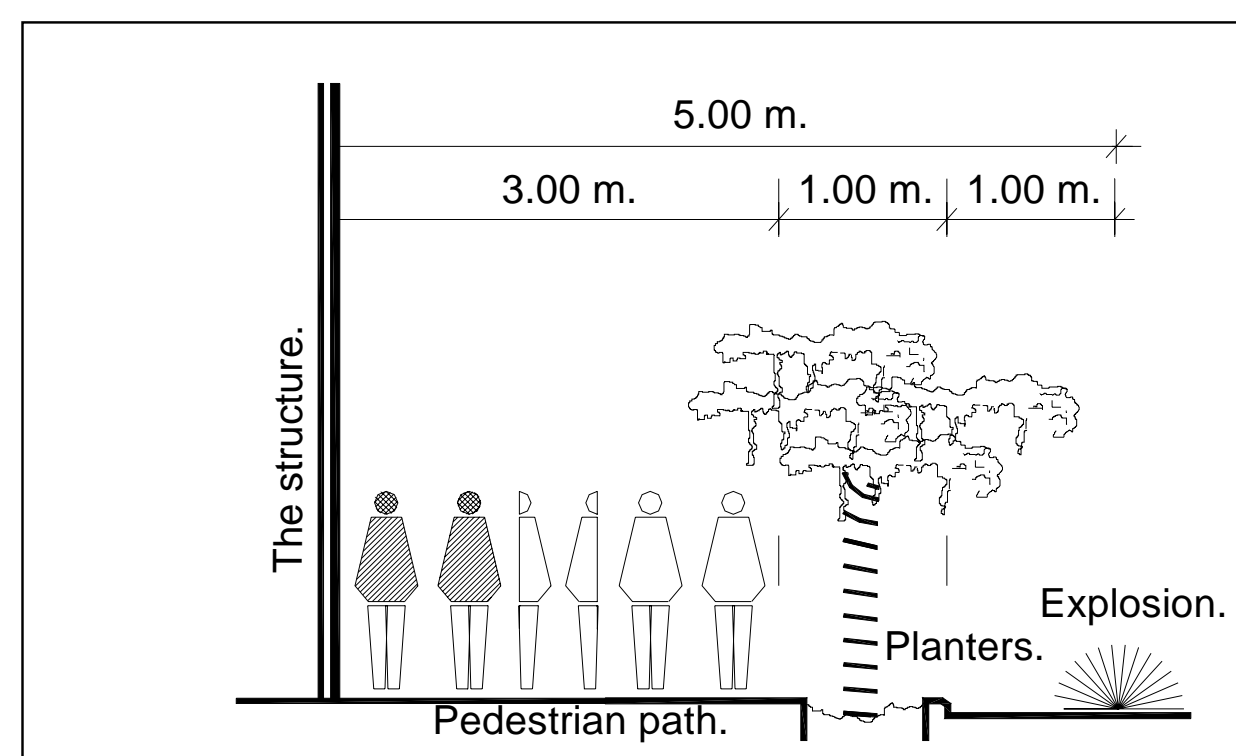


Fig. 4 Distance of the structure from the blast

\section{Target structure height}

The simulations are based around a 25m high, 8 - story reinforced concrete structure. The first story is $4 \mathrm{~m}$ high, all others are $3 \mathrm{~m}$ high. These characteristics were chosen to be representative a typical structure in this category.

\section{Dotted elevation configuration}

Variants of the dotted elevation form, were identified for the test simulations [figure 5]:

Convex dotted elevation.

- Concave dotted elevation.

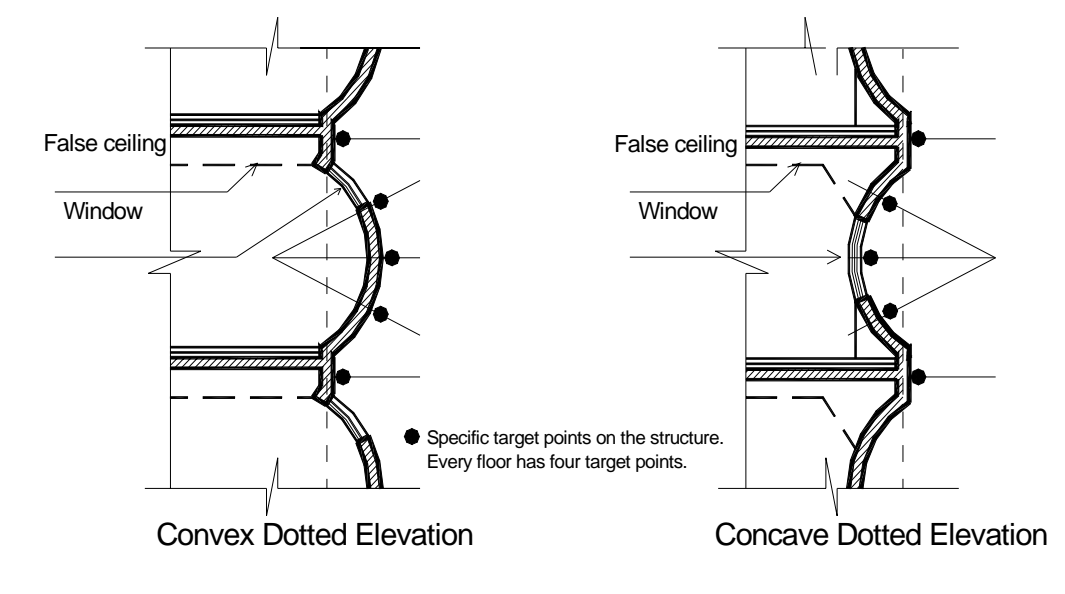

Fig. 5 Dotted Elevation variants

\section{SIMULATIONS RESULTS}

\section{Relative merits of convex dotted elevations}

Figure 6 shows the variation in change of specific impulse and pressure values compared with those of the reference scenario. The curves indicate that the convex dotted elevation form gives better reduction for impulse and pressure values than the concave dotted elevation form. The concave dotted elevation form acts as a collector for the blast waves whereas the convex form tends to disperse the blast waves. 
6.1 The change in impulse values (\%) vs specific target points on structure of the test simulations.

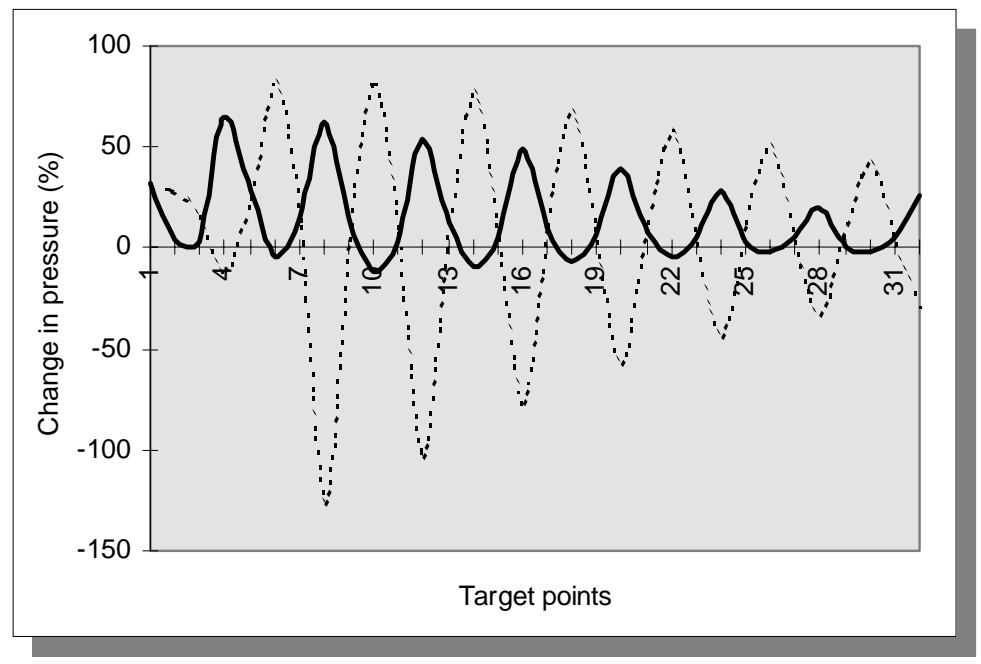

6.2 The change in pressure values (\%) vs specific target points on structure of the test simulations.

\section{DISCUSSION}

Convex dotted elevation

Concave dotted elevation

Four factors favor the use of a convex dotted elevation form to offer a reduction in the effects of blast waves on structures.

\section{The aesthetic view}

It is important that the provision of protection to this category of civilian buildings should not result in an austere appearance.

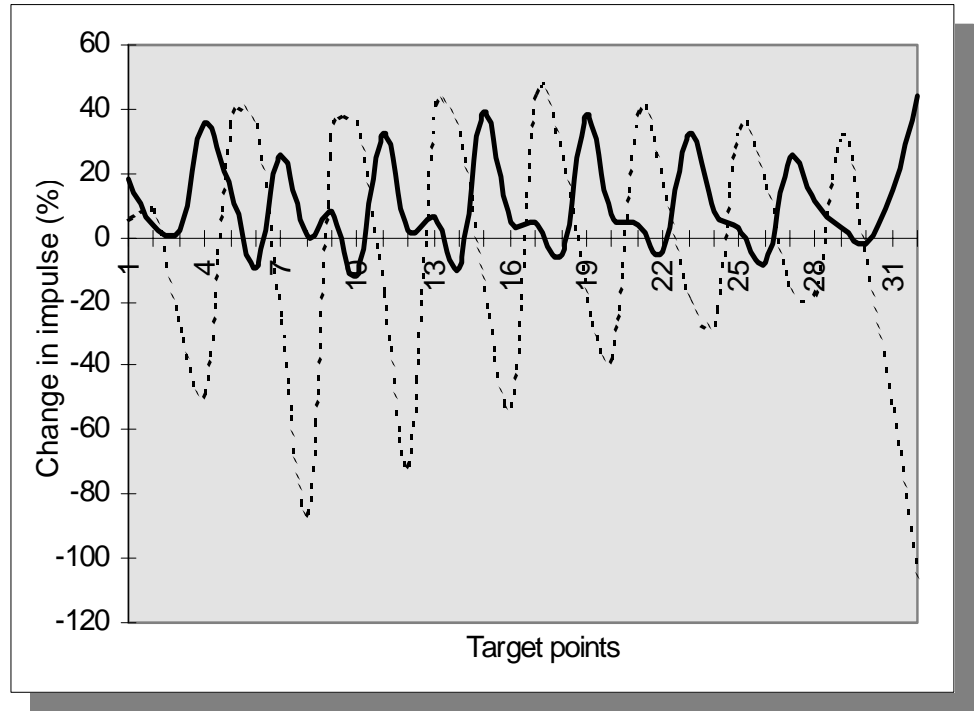




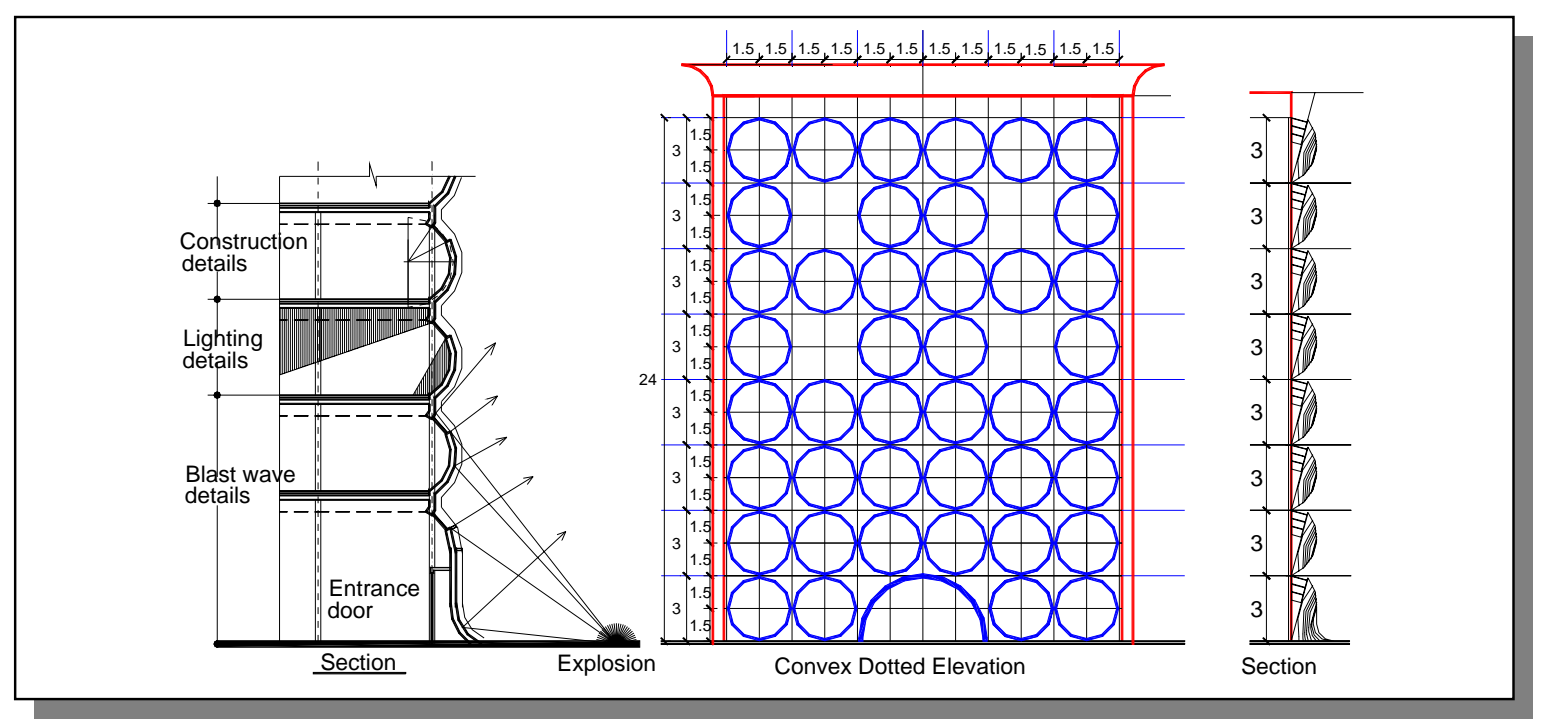

Fig. 7 Architecture view of Convex dotted Elevation Technique The convex dotted elevation form can provide an aesthetically pleasing form for such buildings whilst offering a significant degree of protection. Fig. $7 \& 8$.

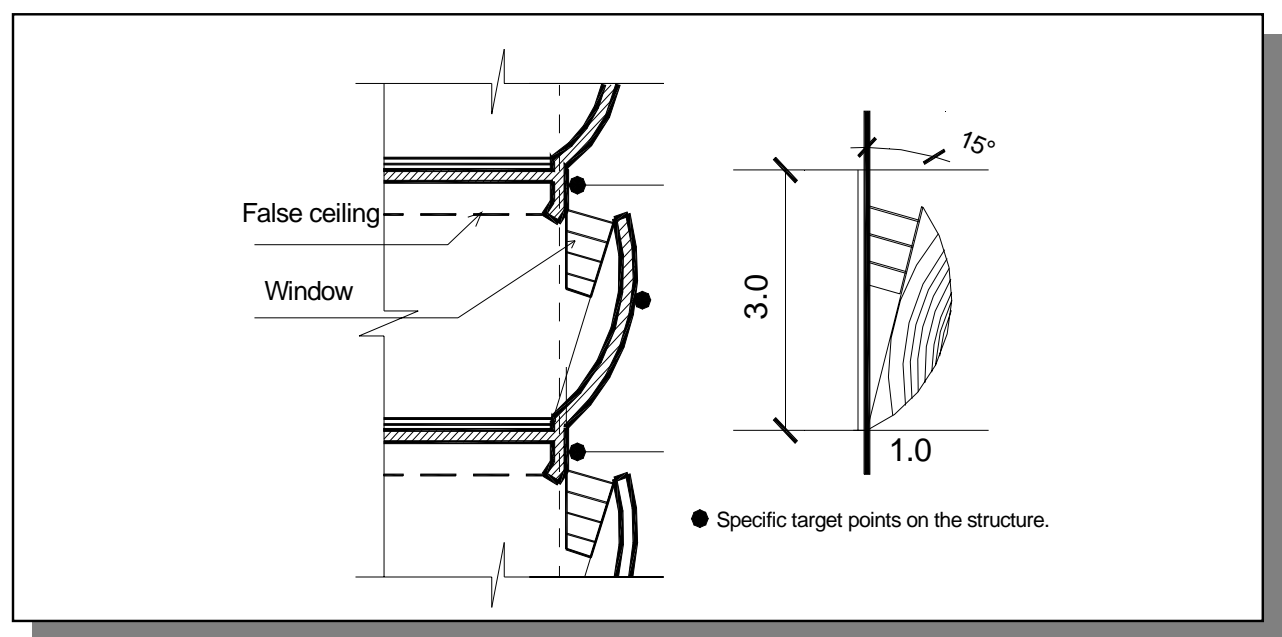

Fig. 8 A detailed view for the dotted elevation technique.

\section{Fragment shielding}

The Fragments of explosion and window glass are a major source of injuring. The convex dotted elevation form can offer a significant reduction of these fragments by suitable location of the building' windows. [4] 


\section{The Natural light}

Figure 9 shows how the convex dotted elevation technique can offer protection without affecting the influx of natural light.

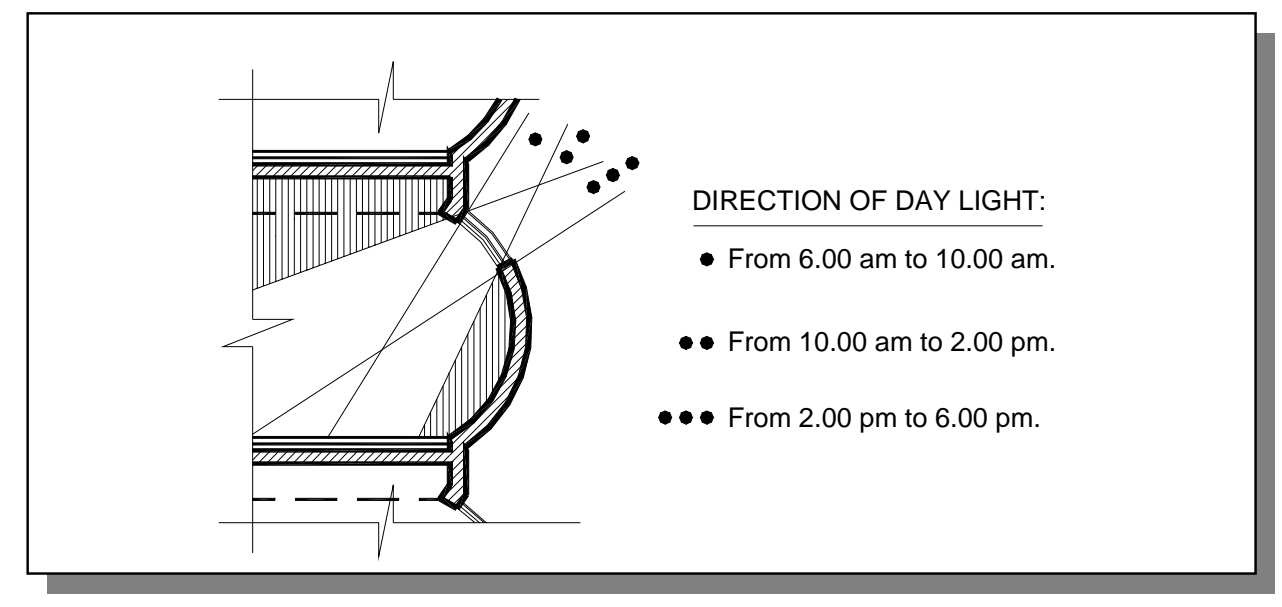

Fig. 9 Influx of natural light

\section{Robustness}

The convex reinforced concrete structural elements will be inherently strong in compression, proving robust against blast loading. [4]

\section{CONCLUSIONS}

1. The convex dotted elevation technique can offer a significant reduction in the effects of blast waves on the structure.

2. The convex dotted elevation form gives better reduction for impulse and pressure values than the concave dotted elevation form.

3. The convex dotted elevation form can be used without affecting the functionality of the structure or the street.

4. The convex dotted elevation form offers benefits of shielding and robustness whilst having good aesthetic appeal and other architectural advantages such as good natural lighting. 


\section{REFERENCE}

[1] AUTODYN 2D \& 3D V4, Century Dynamics Limited, 2002.

[2] ConWep, U.S. Army Engineer Waterways Experiment Station, 1991.

[3] Ehab Hanafi Mahmoud, 3rd International Conference on SHOCK \& IMPACT LOADS ON STRUCTURES, Singapore, 1999.

[4] M. A. Barakat, An architectural approach towards reducing the effects of blast waves and fragments on structure, Melbourne, Australia, 1997. 